Collisionality and safety factor scalings of $\mathrm{H}$-mode energy transport in the MAST spherical tokamak

This content has been downloaded from IOPscience. Please scroll down to see the full text. 2011 Nucl. Fusion 51073045

(http://iopscience.iop.org/0029-5515/51/7/073045)

View the table of contents for this issue, or go to the journal homepage for more

Download details:

IP Address: 150.203.179.67

This content was downloaded on 04/08/2014 at 06:52

Please note that terms and conditions apply. 


\title{
Collisionality and safety factor scalings of H-mode energy transport in the MAST spherical tokamak
}

\author{
M. Valovič ${ }^{1}$, R. Akers ${ }^{1}$, M. de Bock ${ }^{1}$, J. McCone ${ }^{1}$, L. Garzotti ${ }^{1}$, \\ C. Michael ${ }^{1}$, G. Naylor ${ }^{1}$, A. Patel ${ }^{1}$, C.M. Roach ${ }^{1}$, R. Scannell ${ }^{1}$, \\ M. Turnyanskiy ${ }^{1}$, M. Wisse ${ }^{4}$, W. Guttenfelder ${ }^{2}$, J. Candy $^{3}$ and the \\ MAST team ${ }^{1}$ \\ ${ }^{1}$ EURATOM/CCFE Fusion Association, Culham Science Centre, Abingdon, OX14 3DB, UK \\ ${ }^{2}$ Princeton Plasma Physics Laboratory, Princeton University, Princeton, NJ 08543, USA \\ ${ }^{3}$ General Atomics, PO Box 85608, San Diego, CA 92186-5608, USA \\ ${ }^{4}$ University of Basel, Klingelbergstrasse 82, CH-4056, Basel, Switzerland \\ E-mail: martin.valovic@ccfe.ac.uk
}

Received 21 December 2010, accepted for publication 25 May 2011

Published 22 June 2011

Online at stacks.iop.org/NF/51/073045

\begin{abstract}
A factor of 4 dimensionless collisionality scan of H-mode plasmas in MAST shows that the thermal energy confinement time scales as $B \tau_{E \text {,th }} \propto \nu_{* \mathrm{e}}^{-0.82 \pm 0.1}$. Local heat transport is dominated by electrons and is consistent with the global scaling. The neutron rate is in good agreement with the $\nu_{*}$ dependence of $\tau_{E \text {,th }}$ The gyrokinetic code GYRO indicates that micro-tearing turbulence might explain such a trend. A factor of 1.4 dimensionless safety factor scan shows that the energy confinement time scales as $B \tau_{E \text {,th }} \propto q_{\mathrm{eng}}^{-0.85 \pm 0.2}$. These two scalings are consistent with the dependence of energy confinement time on plasma current and magnetic field. Weaker $q_{\text {eng }}$ and stronger $\nu_{*}$ dependences compared with the IPB98y 2 scaling could be favourable for an ST-CTF device, in that it would allow operation at lower plasma current.
\end{abstract}

(Some figures in this article are in colour only in the electronic version)

\section{Introduction}

The first application of spherical tokamaks (STs) is foreseen to be an intense fusion volume neutron source. An example of such a device is the Component Test Facility (ST-CTF) [1], the relevance of which to the fusion energy programme was noted in [2]. So far the extrapolations to the ST-CTF were performed using the IPB98y 2 confinement scaling derived from an international multi-machine database containing only conventional tokamaks [3]. This scaling is, however, not in line with data from STs MAST [4] and NSTX [5, 6], which display a stronger dependence of energy confinement time on toroidal magnetic field and a weaker dependence on plasma current than the IPB98y 2 scaling. Data from STs have been later supplied to the multi-machine tokamak database, now maintained by the International Tokamak Physics Activity (ITPA), and their effect on confinement scaling has been analysed [7,8]. As a continuation of these activities the ITPA group on Transport and Confinement opened a special task TC-12 ('transport and confinement at low aspect ratio') in order to analyse the confinement in STs in more detail.
Typically scalings of energy confinement time are expressed in terms of engineering parameters such as plasma current, magnetic field, etc. These scaling laws are important for their practicality. However, for better insight into underlying physics and the validity of extrapolations plasma physics dimensionless variables should be used instead. In this paper we analyse the scalings of heat transport using two such parameters: normalized collisionality and safety factor. The motivation for selecting these two parameters is discussed in section 2 . The collisionality scaling is determined in section 3 . The safety factor scaling is deduced in section 4 . Discussion and implication of scalings for future devices are described in section 5 .

\section{Gap analysis}

To identify the gaps between existing MAST data and the future ST-CTF device we use the present MAST H-mode dataset that was submitted to the ITPA confinement database and data described later in this paper. The MAST data span the following space of engineering parameters: plasma 

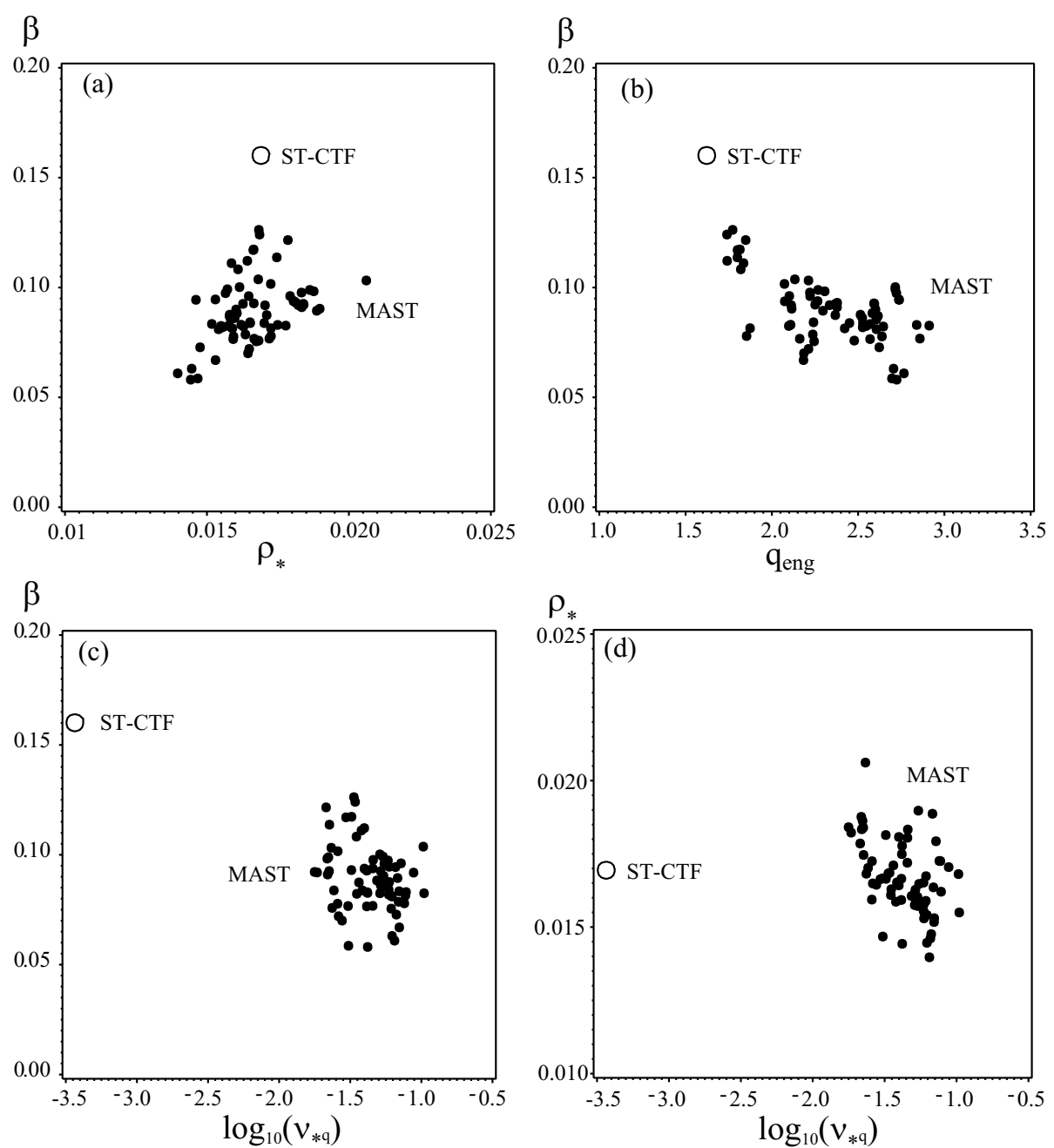

Figure 1. Scatter plots of MAST data in the space of global dimensionless plasma physics parameters. Full symbols: data submitted to ITPA database and data in this paper. Open symbols: position of the ST-CTF device [1]. For MAST data the toroidal beta $\beta=\beta_{\text {MHD }}$ (from MHD equilibrium) and for ST-CTF $\beta=\beta_{\text {th }}$ (only thermal component).

current $I_{\mathrm{p}}=(0.59-1.13) \mathrm{MA}$, major radius at the geometric axis $R=(0.77-0.88) \mathrm{m}$, minor radius $a=(0.50-0.62) \mathrm{m}$, elongation $\kappa=1.6-2.1$, triangularity $\delta=0.3-0.5$, vacuum toroidal field at the geometric radius $B=(0.34-0.50) \mathrm{T}$ and line-averaged density $\bar{n}_{\mathrm{e}}=(2.1-5.1) \times 10^{19} \mathrm{~m}^{-3}$. Total injected NBI power is $P_{\mathrm{NBI}}<3.5 \mathrm{MW}$ and the energy of the neutral beams is $E_{\mathrm{NBI}}<65 \mathrm{keV}$. Beams are injected in the direction of the plasma current. In our studies plasmas have a double null divertor configuration with deuterium as a working gas. Sawteeth are generally avoided by application of neutral beam heating (NBI) during current ramp-up. The data are mapped to the space of global dimensionless parameters, such as toroidal $\beta$, normalized Larmor radius $\rho_{*}=(M T)^{1 / 2} /(a B)$, engineering safety factor $q_{\mathrm{eng}}=2 \pi a^{2} \kappa B /\left(R \mu_{0} I_{\mathrm{p}}\right)$ and normalized collisionality $v_{* q} \propto \bar{n}_{\mathrm{e}} T^{-2} R q_{\mathrm{eng}}(R / a)^{3 / 2}$. Here $T=W_{\text {th }} /\left(3 V \bar{n}_{\mathrm{e}}\right)$ is the volume-averaged temperature, $M$ is the ion mass, $V$ is the plasma volume and $W_{\text {th }}$ is the thermal energy content. The parameters for the ST-CTF device are the following [1]: $I_{\mathrm{p}}=6.5 \mathrm{MA}, R=0.85 \mathrm{~m}, a=0.55 \mathrm{~m}$, $\kappa=2.4, B=2.5 \mathrm{~T}, \bar{n}_{\mathrm{e}}=1.2 \times 10^{20} \mathrm{~m}^{-3}$, normalized beta $\beta_{\mathrm{N}}=3.5$ and $H=1.3$ is the enhancement relative to IPB98 22 confinement scaling [3].
Inspection of the scatter plots in figures $1(a)-(c)$ shows that the largest gap in extrapolating MAST data to the ST-CTF device exists in the normalized collisionality. This gap is about one and a half orders of magnitude and thus even a small error in the scaling of energy confinement time with collisionality means a large prediction error. The gap in safety factor is quite modest. However, MAST plasmas with $q_{\text {eng }}$ as chosen for ST-CTF have control difficulties, such as low ELM frequency and short pulse length. Therefore, it might be desirable to increase $q_{\text {eng }}$ in the ST-CTF operation point to reduce the uncertainty of the extrapolation. Whether such a change is possible will depend on the scaling of confinement with safety factor. Another reason to treat collisionality and safety factor scalings simultaneously is that these two parameters determine the scalings with plasma current and toroidal magnetic field as discussed in [4].

As already mentioned the data in figure 1 are made of larger, less controlled dataset, and thus the gap analysis based solely on figures $1(a)-(c)$ could be distorted by collinearities between variables. Most notably anti-correlation between $\rho_{*}$ and $v_{* q}$ might be expected due to their dependences on temperature. If true, the gap between MAST and ST-CTF 
Table 1. Parameters of $v_{*}$ scan.

\begin{tabular}{lcc}
\hline Shot number & 22769 & 22664 \\
\hline Time $(\mathrm{s})$ & 0.20 & 0.23 \\
$a(\mathrm{~m})$ & 0.57 & 0.58 \\
$R(\mathrm{~m})$ & 0.813 & 0.816 \\
$\kappa$ & 2.0 & 2.0 \\
$\delta$ & 0.42 & 0.45 \\
$B(\mathrm{~T})$ & 0.34 & 0.50 \\
$I_{\mathrm{p}}(\mathrm{kA})$ & 592 & 886 \\
$\bar{n}_{\mathrm{e}}\left(10^{19} \mathrm{~m}^{-3}\right)$ & 3.2 & 3.3 \\
$q_{\mathrm{eng}}$ & 2.3 & 2.3 \\
$W_{\text {th }}(\mathrm{kJ})$ & 41 & 87 \\
$W_{\mathrm{e}}(\mathrm{kJ})$ & 20 & 40 \\
$P_{\mathrm{NBI}}(\mathrm{MW})$ & 3.0 & 3.2 \\
\hline
\end{tabular}

along $v_{* q}$ estimated from figure $1(c)$ will be based on data with the largest $\rho_{*}$ and not those with the same $\rho_{*}$ values as for ST-CTF, i.e. the gap along $\nu_{* q}$ will be underestimated. Figure $1(d)$, however, shows that the collinearity between $\rho_{*}$ and $v_{* q}$ is relatively small and does not distort the gap analysis significantly.

To evaluate the aforementioned scalings let us write the thermal energy confinement time in a conventional power law form:

$$
\tau_{E, \mathrm{th}} B \propto \rho_{*}^{x_{\rho}} \beta^{x_{\beta}} v_{*}^{x_{v}} q_{\mathrm{eng}}^{x_{q}} \kappa^{x_{\kappa}} .
$$

Here collisionality is defined as $\nu_{*} \propto \bar{n}_{\mathrm{e}} T^{-2} R$, i.e. without the safety factor. This choice of collisionality definition is identical to that used in the $v_{*}$ scan in DIII-D [9], and thus allows a direct comparison between both machines. The main reason for this choice is to keep $q_{\text {eng }}$ dependence in scaling (1) fully explicit as the heat transport can depend on $q_{\text {eng }}$ independent of $v_{*}$. As shown below, the exponent $x_{v}$ is determined from the scan, where $q_{\text {eng }}$ is kept constant. Therefore, the result should be independent of the definition of $v_{*}$ as well as the prediction to future devices. The definition of $\beta$ in scaling (1) contains only a thermal component; $\beta=\beta_{\mathrm{th}}$.

\section{Collisionality scan of energy transport}

The exponent $x_{v}$ in the scaling formula (1) can be determined from a set of plasmas which differ in collisionality $v_{*}$ but with the other dimensionless parameters kept constant. From the requirement that $\rho_{*} \propto \sqrt{T} / B=$ const, $\beta \propto \bar{n}_{\mathrm{e}} T / B^{2}=$ const and $q \propto B / I_{\mathrm{p}}=$ const one finds that the plasma density, temperature and plasma current should depend on toroidal magnetic field as $\bar{n}_{\mathrm{e}} \propto B^{0}, T \propto B^{2}$ and $I_{\mathrm{p}} \propto B$. The range of the scan is controlled by the span of the toroidal magnetic field, as $v_{*} \propto n / T^{2} \propto B^{-4}$. In MAST, for fixed major radius of $R=0.81 \mathrm{~m}$, the maximum toroidal field is $B_{\max }=0.50 \mathrm{~T}$. The lower point was set empirically to $B_{\min }=0.34 \mathrm{~T}$ so that the beam deposition is not significantly affected by unconfined orbits. (Note that in STs the banana width is approximately equal to the Larmor radius and thus toroidal magnetic field has the same affect as plasma current in controlling fast ion losses.) This choice provides a collisionality scan by a factor of $\left(B_{\max } / B_{\min }\right)^{4}=4.6$.

\subsection{Matching dimensionless parameters}

Table 1 shows the parameters of two discharges that have been arranged to satisfy the requirements for the collisionality scan.
The first parameter to match is the plasma density. Due to the dependence of the $\mathrm{L}-\mathrm{H}$ threshold on the magnetic field, the plasma with lower $B$ enters $\mathrm{H}$-mode at lower density than its higher field counterpart. In addition, after transition to $\mathrm{H}$-mode the density control is limited. Therefore, to match the density, the time slice for high $B$ plasma has to be selected at the beginning of the $\mathrm{H}$-mode phase where the contribution of the change of energy content to the power balance is substantial. Table 1 shows that the line-averaged density has been matched within $\pm 1.5 \%$. The density profiles are flat, as is typical for ELMy H-mode, and the profile matching quality is shown in figure $2(a)$.

The second parameter to adjust is the plasma temperature. This is done by tuning the neutral beam power, mainly by varying beam current. Table 1 shows that the ratio of thermal energy content $W_{\text {th }}$ along the scan is 2.1, in good agreement with the required value of $\left(B_{\max } / B_{\min }\right)^{2}=$ 2.16. Such a match is achieved by a difference of $10 \%$ in beam power. Here, $W_{\text {th }}$ is calculated from the electron density $n_{\mathrm{e}}$ and electron temperature $T_{\mathrm{e}}$ measured by Thomson scattering, ion temperature $T_{\mathrm{i}}$ measured by charge exchange recombination spectroscopy (CXRS), effective charge $Z_{\text {eff }}$ by bremsstrahlung emission and plasma shape from equilibrium reconstruction (EFIT) constrained by motional Stark effect (MSE) measurements. $Z_{\text {eff }}$ data are complemented by measurement of carbon concentration using CXRS. Table 1 shows that the ratio of electron to ion temperatures is not exactly constant along the scan. Variation of electron energy content $W_{\mathrm{e}}$ along the scan is 2.0 while the variation of ion energy content $W_{\mathrm{i}}=W_{\mathrm{th}}-W_{\mathrm{e}}$ is 2.2. In other words, the electron temperature varies somewhat less, while the ion temperature varies more than required for an ideal scan. This is also seen from the electron and ion temperature profiles shown in figures $2(b)$ and $(c)$, where an ideal match is indicated by dotted lines. The shape of the $T_{\mathrm{e}}$ profile is preserved along the scan but the profile of $T_{\mathrm{i}}$ becomes more peaked at lower collisionality. In order to keep the $T_{\mathrm{i}} / T_{\mathrm{e}}$ ratio constant along the scan a separate heating system would be required and this is not yet available on MAST. As a result of changing $T_{\mathrm{i}} / T_{\mathrm{e}}$ the range of the $v_{*}$ scan depends on which temperature is used in the definition of volume-averaged collisionality. The collisionality as defined using the electron temperature, $v_{* \mathrm{e}} \propto \bar{n}_{\mathrm{e}}^{3} / W_{\mathrm{e}}^{2}$, varies by a factor of 3.6, while using the thermal energy, $v_{*} \propto \bar{n}_{\mathrm{e}}^{3} / W_{\text {th }}^{2}$, results in a variation by a factor of 4.1 .

A small deviation in electron temperature from its ideal value translates into a variation of the toroidal beta and normalized Larmor radius along the scan. Measured by volume-averaged electron temperature, these variations are within $10 \%: \beta_{\mathrm{e}, 0.5 \mathrm{~T}} / \beta_{\mathrm{e}, 0.34 \mathrm{~T}}=0.92$ and $\rho_{* \mathrm{e}, 0.5 \mathrm{~T}} / \rho_{* \mathrm{e}, 0.34 \mathrm{~T}}=$ 0.96. In tokamaks the $\beta$ dependence is typically bounded by the exponent in the range of $x_{\beta} \in(-1,0)$ while the $\rho_{*}$ dependence is typically described by gyro-Bohm scaling with $x_{\rho} \approx-3$. Therefore the mismatch in $\rho_{*}$ is more significant. The error on the $v_{*}$ exponent due to $\rho_{*}$ mismatch

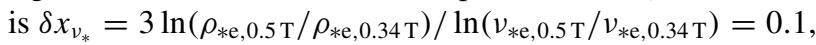
and its direction is such that it makes the $x_{v}$ exponent less negative.

The profiles of safety factor $q$ are well matched in the outer half of the plasma (see figure $2(d)$ ). However, the high $v_{*}$ shot has $q<1$ in the plasma centre and as a result sawteeth 

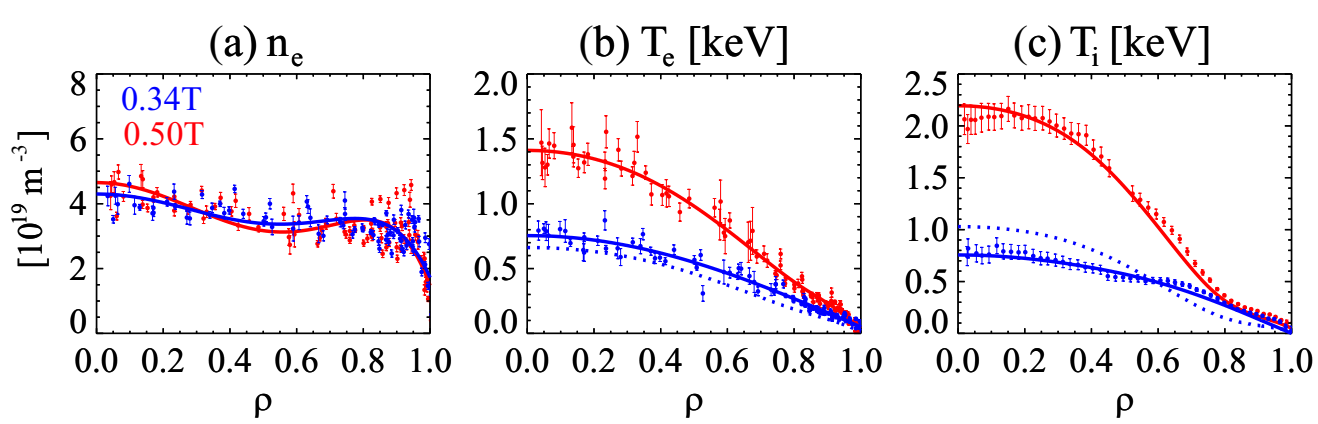

(d) $\mathrm{q}, \mathrm{M}_{\mathrm{tor}} \mathrm{x} 10$

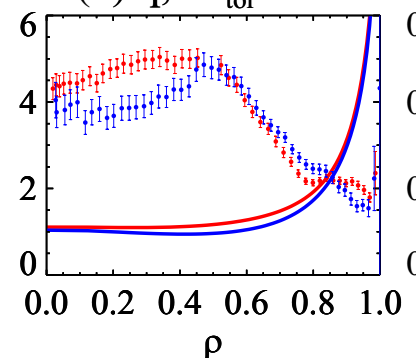

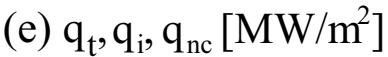

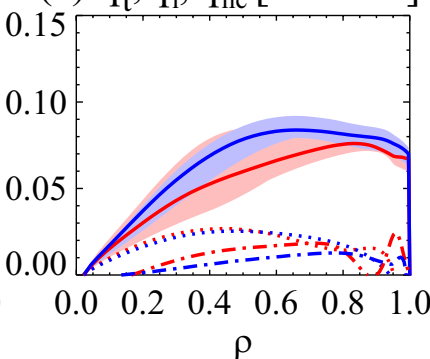

(f) $\chi_{e, 0.5 \mathrm{~T}} / \chi_{e, 0.34 \mathrm{~T}}$

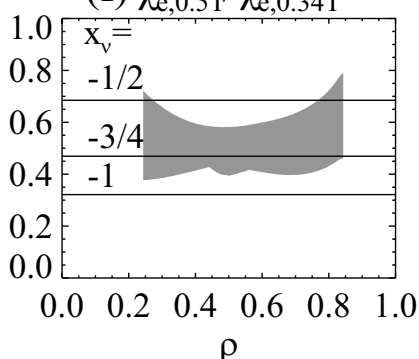

Figure 2. Profiles in collisionality scan in table 1 (\#22769-blue symbols, \#22664-red symbols). (a) Electron density $n_{\mathrm{e}} ;(b)$ electron temperature $T_{\mathrm{e}} ;(c)$ ion temperature $T_{\mathrm{i}}$. Dotted lines show an ideal match. $(d)$ Discrete symbols: toroidal Mach number

$M_{\text {tor }}=V_{\text {tor }} /\left(T_{\mathrm{i}} / m_{\mathrm{i}}\right)^{1 / 2}$, where $m_{\mathrm{i}}$ is the deuterium ion mass; solid lines: $q$ profiles. $(e)$ Solid lines: total heat flux $q_{\mathrm{t}}$, dotted lines: ion heat flux $q_{\mathrm{i}}$, dashed-dotted lines: ion neoclassical heat flux $q_{\mathrm{nc}}$. $(f)$ Shaded area: ratio of electron heat diffusivities $\chi_{\mathrm{e}, 0.5 \mathrm{~T}} / \chi_{\mathrm{e}, 0.34 \mathrm{~T}}$, lines show the ratios expected from different scaling exponents. $\rho=\psi_{\mathrm{N}}^{0.5}$, where $\psi_{\mathrm{N}}$ is the normalized poloidal magnetic flux. Shaded areas in $(e)$ and $(f)$ represent the data scatter within $\pm 5 \mathrm{~ms}$ time window.

are present in this plasma. The toroidal Mach number $M_{\mathrm{tor}}$ is similar for both shots, as seen in figure $2(d)$.

\subsection{Validation of power loss}

When dimensionless parameters are matched the collisionality exponent is determined by the change in thermal power loss along the scan: $P_{\mathrm{L}, \text { th }} \propto v_{*}^{-x_{v}-3 / 4}$. The thermal power loss $P_{\mathrm{L}, \mathrm{th}}$ is calculated by the TRANSP code ${ }^{4}$ with the help of a data pre-processor. The number of test particles in TRANSP Monte-Carlo simulations of beam ions varied between $10^{3}$ to $10^{4}$ with no significant difference in the calculated heating power. Better convergence is sometimes observed when more test particles are used, probably due to reduced numerical noise. Special attention has to be paid to the distribution of power between full, half and third energy components because the beam power was adjusted mainly by beam current. Energy components are calculated from actual beam perveance using calibration tables. With classical dynamics of fast ions the calculated neutron emission is higher than the values measured by a fission chamber. Simultaneously the fast ion energy content is larger than found by the EFIT code constrained by MSE. These differences are attributed to the anomalous loss of fast ions. In TRANSP these losses are modelled by a diffusion with coefficient $D_{\text {fast }}$. Good agreement between the calculated and measured neutron rate is found with $D_{\text {fast }}=2 \mathrm{~m}^{2} \mathrm{~s}^{-1}$ and $3 \mathrm{~m}^{2} \mathrm{~s}^{-1}$ for the low and high $v_{*}$ points, respectively. Such values of $D_{\text {fast }}$ result in approximately the same total heat flux $q_{\mathrm{t}}=q_{\mathrm{e}}+q_{\mathrm{i}}$ for both shots in the scan, as seen in figure 2(e). This is also reflected in the global power loss, which is similar for both shots $P_{\mathrm{Lth}, 0.34 \mathrm{~T}} / P_{\mathrm{Lth}, 0.50 \mathrm{~T}}=1.05$. Such a value

4 TRANSP code, http://w3.pppl.gov/transp/. with $v_{* \mathrm{e}, 0.34 \mathrm{~T}} / \nu_{* \mathrm{e}, 0.50 \mathrm{~T}}=3.6$ gives the collisionality scaling $\tau_{\mathrm{E}, \mathrm{th}} B \propto \nu_{* \mathrm{e}}^{-0.79}$.

The values of fast ion diffusivities mentioned above might look rather large. It should be noticed, however, that these losses can play the role in the balance of fast ion content only if corresponding loss time $\tau_{\mathrm{D}} \propto 1 / D_{\text {fast }}$ is comparable to the collisional slowing down time $\tau_{\mathrm{D}} \sim \tau_{\mathrm{sd}}$. In our plasmas the collisional slowing down times are quite short due to relatively low electron temperatures $\left(\tau_{\mathrm{sd}} \propto T_{\mathrm{e}}^{3 / 2}\right.$ ) and this explains why rather large values of anomalous fast ion diffusion coefficients are required to modify fast ion content in calculations.

Figure 2(e) shows that most of the heat flows along the electron channel while the ion heat flux is close to the neoclassical level. For this reason only the electron heat flux was analysed in more detail. Figure $2(f)$ shows the ratio of electron heat diffusivities $\chi_{\mathrm{e}, 0.5 \mathrm{~T}} / \chi_{\mathrm{e}, 0.34 \mathrm{~T}}$ along the scan. This value is about 0.5 so that the collisionality dependence can be bracketed by $\chi_{\mathrm{e}} B^{-1} \propto v_{* \mathrm{e}}^{1 / 2}$ to 1 . This is in good agreement with the global energy confinement time scaling.

The robustness of the scaling from the two-point scan was tested by adding more data points. The results are a dataset with larger span along the collisionality while variations of $\rho_{*}$, $\beta$ and $q_{\text {eng }}$ are kept within $20 \%$, as shown in the top panel of figure 3. Log-linear regression ${ }^{5}$ of the extended dataset of plasmas with shapes similar to that in table $1(\kappa=2.0)$ provides the scaling, $\tau_{E, \mathrm{th}} B \propto \nu_{* \mathrm{e}}^{-0.82}$ with standard error on the exponent $\delta x_{v_{*}}=0.1$. This is similar to the result from the two-point scan. Data with lower elongation $(\kappa=1.7)$ show a similar trend.

5 1999-2001 PROC REG, SAS/STAT ${ }^{\circledR}$ Software: Release 9.1 SAS Institute Inc. 


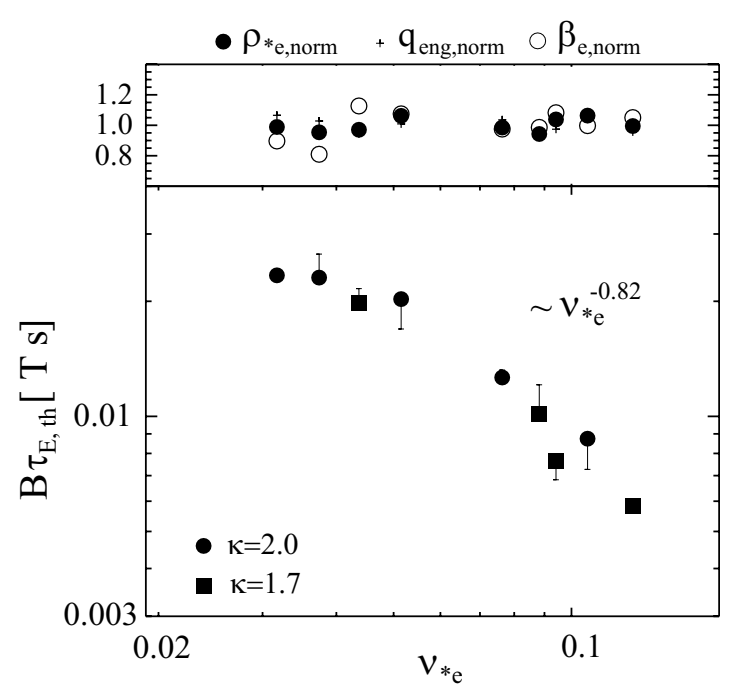

Figure 3. Collisionality scan of thermal energy confinement time. Vertical bars show the size of the correction due to variations in $\rho_{* e}$, assuming gyro-Bohm scaling. $\nu_{* \mathrm{e}} \propto \bar{n}_{\mathrm{e}}\left\langle T_{\mathrm{e}}\right\rangle^{-2} R$,

$\left\langle T_{\mathrm{e}}\right\rangle=W_{\mathrm{e}} /\left(1.5 V \bar{n}_{\mathrm{e}}\right), V$ is the plasma volume. Top panel: variations of electron Larmor radius, beta and $q_{\text {eng }}$, all normalized to average values along the scan. The scaling is based only on $\kappa=2.0$ data.

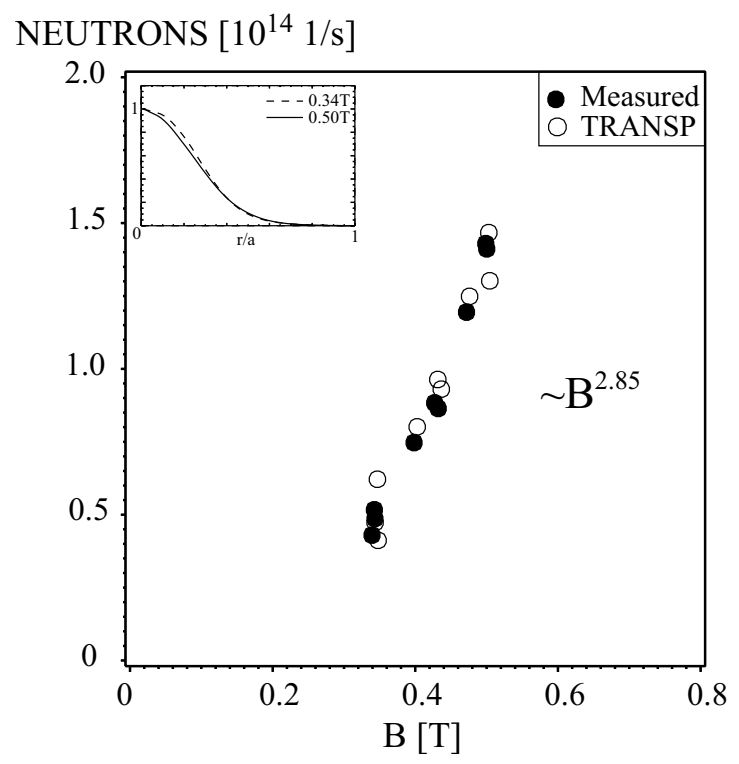

Figure 4. Toroidal field dependence of the neutron rate along the collisionality scan. Full symbols are measured and open symbols are calculated by TRANSP. The dataset is the same as in figure 3 . Regression is on measured data only. The inset shows the normalized profiles of neutron emission from TRANSP for the shots in table 1 .

\subsection{Effect of fast ion losses}

As already mentioned the scaling depends on a model for fast ion losses. Here we used a spatial diffusion coefficient $D_{\text {fast }}$, which is constant along minor radius and is energy independent. The value $D_{\text {fast }}$ is varied until the calculated neutron rate equals the measured value. The quality of this match in shown in figure 4.

If our fast ion model is incorrect and, for example, the smaller measured neutron rate is due to enhanced diffusion in velocity space or due to very localized spatial diffusion in the core, then the energy of fast ions is not lost and the scaling will change. It is therefore useful to estimate the sensitivity of the scaling exponent to the fast ion model. For the scan in table 1 inclusion of fast ion losses decreases the power loss by a factor of 1.4. This correction is, however, the same for both the low and high $v_{*}$ points so that the ratio of power loss $P_{\mathrm{th}, 0.34 \mathrm{~T}} / P_{\mathrm{th}, 0.5 \mathrm{~T}}$ remains unchanged (within 3\%). As a result the exponent $x_{v}$ is relatively insensitive to the fast ion loss model.

It is outside the scope of this paper to study the mechanism of fast ion losses. Here we note that for the low field shot in table 1 (and figure 4) clear drops in neutron rate are seen during sawteeth. For all other shots in figure 4 sawteeth are absent and the drops in neutron rate could be correlated with bursts of fishbones. These observations suggest that MHD events could control the fast ion losses; however, detailed analysis is required to quantify these effects. From the point of view of heat transport it is important to quantify whether fast ions are lost or just redistributed towards the outer part of the plasma where temperature is lower, leading to a shorter slowing down time and lower neutron rate. A limited number of TRANSP runs with a box-shaped profile of $D_{\text {fast }}$ localized in $r / a<0.5$ did not lead to a convincingly better fit to the measured neutron rate and total energy content simultaneously. Relatively low power in the divertor, measured by infrared camera, indicates that fast ions might indeed be lost, however, this has yet to be quantified. New diagnostics, such as a fast ion $D_{\alpha}$ emission camera and neutron detector with spatial resolution, are being commissioned to answer this important question.

\subsection{Neutron emission}

The collisionality scaling can be checked using the dependence of the fusion neutron rate $S_{\mathrm{n}}$ on the toroidal magnetic field. This trend is shown in figure 4 and log-linear regression gives $S_{\mathrm{n}} \propto B^{2.85}$. In the dataset the variation of beam voltage is small (standard deviation $3.8 \%$ from its mean value $E_{\mathrm{NBI}}=$ $62.1 \mathrm{keV})$ and $Z_{\text {eff }}$ in the centre is close to 1 . Therefore the neutron rate, which is dominated by beam-thermal reactions, is $S_{\mathrm{n}} \propto \tau_{\mathrm{sd}} P_{\mathrm{NBI}}$,heat , where $\tau_{\mathrm{sd}}$ is the beam slowing down time and $P_{\mathrm{NBI}, \text { heat }}$ is the dissipated beam power. Ignoring ohmic power and the $\mathrm{d} W_{\text {th }} / \mathrm{d} t$ term, the neutron rate scales with magnetic field as $S_{n} \propto T_{\mathrm{e}}^{3 / 2} P_{\mathrm{L}, \mathrm{th}} \propto T_{\mathrm{e}}^{3 / 2} v_{*}^{-x_{v}-3 / 4} \propto B^{4 x_{v}+6}$. Comparing this with the measured trend one finds that the collisionality exponent is $x_{v}=-0.79$, in good agreement with the previous analysis. The inset in figure 4 shows that the normalized neutron emission profiles, as calculated by TRANSP, can be regarded as self similar so that the profile effects do not play a significant role along the scan.

This method is not fully independent to the analysis in section 3.2 as both methods use the neutron rate data. However, the agreement between these methods is also not trivial as it confirms a very good match of all relevant profiles along the scan, including $Z_{\text {eff }}$.

\subsection{Interpretation of the $v_{*}$ scan}

The GYRO code [10] has been used to analyse the $v_{*}$ scan. Experimental profiles from the high $v_{*}$ plasma in figure 2 


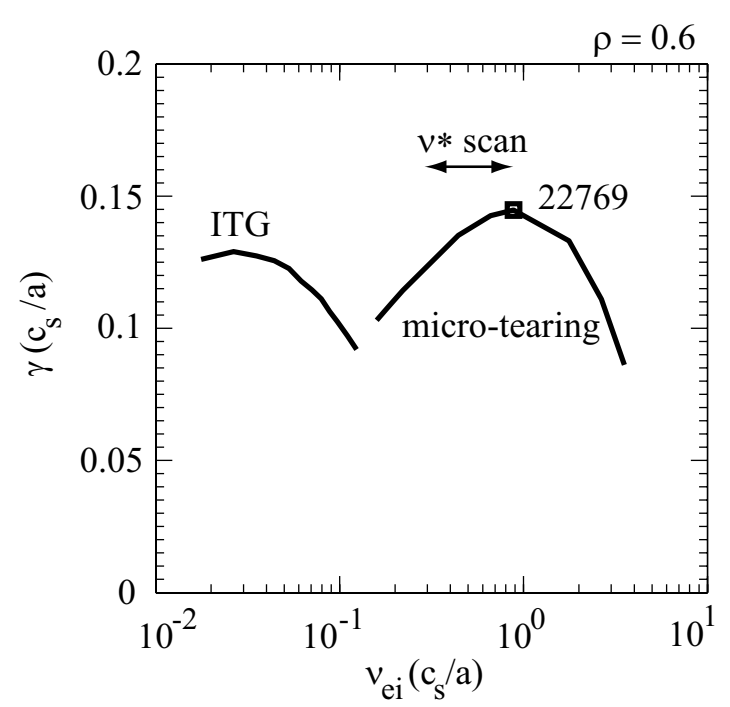

Figure 5. Linear growth rate of micro-tearing and ITG modes calculated by the GYRO code at $\rho=0.6$. The square represents the high $v_{*}$ point in table $1 . v_{\mathrm{ei}}$ is the electron-ion collision frequency.

have been used in the code as a starting point and then the collisionality has been varied numerically. The linear calculations use the EFIT equilibrium, include kinetic ions and electrons, collisionality, fully electromagnetic perturbations (shear and compressional), and have been benchmarked against GS2 [11] calculations. Figure 5 shows that the micro-tearing mode is unstable when using the experimental collisionality. The normalized linear growth rate $\gamma$ decreases as the collisionality is reduced below the high $\nu_{*}$ point in our scan (table 1) consistent with the experimental scaling trend. The possibility for micro-tearing modes having a role in MAST plasmas has been highlighted before [12] and a similar conclusion has been drawn for NSTX [13]. Note, however, that the dependence of $\gamma$ on collisionality in figure 5 is rather weak to explain our $v_{*}$ scaling from a simple mixing length argument at constant wave number. Comprehensive nonlinear simulations of micro-tearing modes are computationally demanding and such work is ongoing [14].

Other ion scale instabilities (ion temperature gradient, ITG, or trapped electron mode, TEM) are also sometimes found to be unstable in MAST. For example, figure 5 shows that at much smaller collisionality the ITG mode can dominate. However, the growth rates are reduced with increasing collisionality as collisions stabilize the trapped electron contributions that enhance the ITG growth rates (e.g. [15]). The fact that ITG is not dominant experimentally is consistent with our observation that ion transport is close to neoclassical in the outer region of the plasma (see figure 2). The electron temperature gradient (ETG) instability is also found to be unstable in many MAST H-mode plasmas [16]. Nonlinear GYRO simulations for the high $v_{*}$ discharge (table 1) find that the ETG transport increases with decreasing collisionality [14]. This is due to enhanced transport at the lowest wavenumbers where the trapped electrons are influenced by collisions.

Collisionality could also affect turbulent transport through other indirect effects, such as the stabilizing influence of plasma rotation, or zonal flows which could themselves be $v_{*}$ dependent. Nonlinear simulations indicate that sheared toroidal flows are usually too weak to significantly suppress the ETG transport in MAST plasmas with $P_{\mathrm{NBI}} \sim 2 \mathrm{MW}$ $[16,17]$. In some strongly rotating plasmas in NSTX with $P_{\mathrm{NBI}} \sim 4 \mathrm{MW}$ the reduction of ETG turbulence, however, has been observed [18]. Nonlinear ETG simulations for our $v_{*}$ scan with $P_{\mathrm{NBI}} \sim 3 \mathrm{MW}$ remain to be performed. Note, however, that along the $v_{*}$ scan, the toroidal Mach number is approximately preserved (figure $2(d)$ ) so that the shearing rate in $c_{\mathrm{s}} / a$ units is also approximately constant. This means that even if shear flow stabilization is active it should not itself produce collisionality dependence.

Finally note that the collisionality dependence could also arise from an accumulated mismatch of other sensitive parameters such as temperature gradients. Another candidate is a combination of profile stiffness and $v_{*}$ dependence of transport in the plasma edge. In addition it is suggested that electron transport could be enhanced by beam driven Alfvén eigenmodes [19], in particular towards high $v_{*}$ where the ratio of beam velocity to Alfvén velocity increases due to a decrease in the toroidal magnetic field. Future work is planned to explore these hypotheses.

\section{4. $q$-scan}

The safety factor exponent $x_{q}$ in scaling (1) has been evaluated using a factor of 1.46 scan of plasma current at constant toroidal magnetic field, electron density and plasma temperature. Figure 6 shows the profile analysis for two plasmas from such a scan. Plasma densities and temperatures are well matched in the pair (figures $6(a)-(c)$ ). The match of thermal energy content is also good: $W_{\text {th }}=69.8-69.9 \mathrm{~kJ}$. Small variations of plasma geometry outside our control mean that $q_{\text {eng }}$ varies by a factor of 1.32, i.e. less than the change of $I_{\mathrm{p}}$ (figure $6(d)$ ).

In order to match the plasma temperature, the heating power has to be adjusted. A plasma with lower plasma current (high $q_{\mathrm{eng}}$ ) needs higher heating power while for a plasma with higher current (low $q_{\text {eng }}$ ) low heating power is sufficient. The exponent of the safety factor in scaling (1) is then related to the thermal power loss as $P_{\mathrm{L}, \mathrm{th}}=W_{\mathrm{th}} / \tau_{E \text {,th }} \propto q_{\mathrm{eng}}^{-x_{q}}$. Similar to the $v_{*}$ scan, anomalous fast ion losses have to be included in TRANSP to match the measured neutron rate. The analysis gives, for the ratio of thermal power loss, $P_{\mathrm{th}, 0.9 \mathrm{MA}} / P_{\mathrm{th}, 0.6 \mathrm{MA}}=$ 0.81. Consequently, the safety factor exponent is found to be $x_{q}=-\ln \left(P_{\mathrm{th}, 0.9 \mathrm{MA}} / P_{\mathrm{th}, 0.6 \mathrm{MA}}\right) / \ln \left(q_{\mathrm{eng}, 0.9 \mathrm{MA}} /\right.$ $\left.q_{\text {eng, } 0.6 \mathrm{MA}}\right)=-0.73$.

The global safety factor scaling is confirmed by a local heat transport analysis. Figures $6(e)$ and $(f)$ show that the ratio of heat flux $q_{\mathrm{t}, 0.9 \mathrm{MA}} / q_{\mathrm{t}, 0.6 \mathrm{MA}}$ is also consistent with the value of $x_{q}$ found from the global confinement time. As was the case in the collisionality scan, most of the heat is transported along the electron channel (figure 6(e)). The ratio of electron heat diffusivities in the outer half of the plasma $\rho \in(0.5,0.9)$ is in the range $\chi_{\mathrm{e}, 0.9 \mathrm{MA}} / \chi_{\mathrm{e}, 0.6 \mathrm{MA}} \in(0.6,0.9)$, while the ratio of local values of the safety factor lie in the range $q_{0.9 \mathrm{MA}} / q_{0.6 \mathrm{MA}} \in(0.75,0.95)$. This would again broadly imply linear scaling of $\chi_{\mathrm{e}}$ with $q$. However, the radial profiles are too complicated to be described as $\chi_{\mathrm{e}} \propto q^{-x_{q}}$ with a single value of $x_{q}$ (see figure $6(f)$ ). 

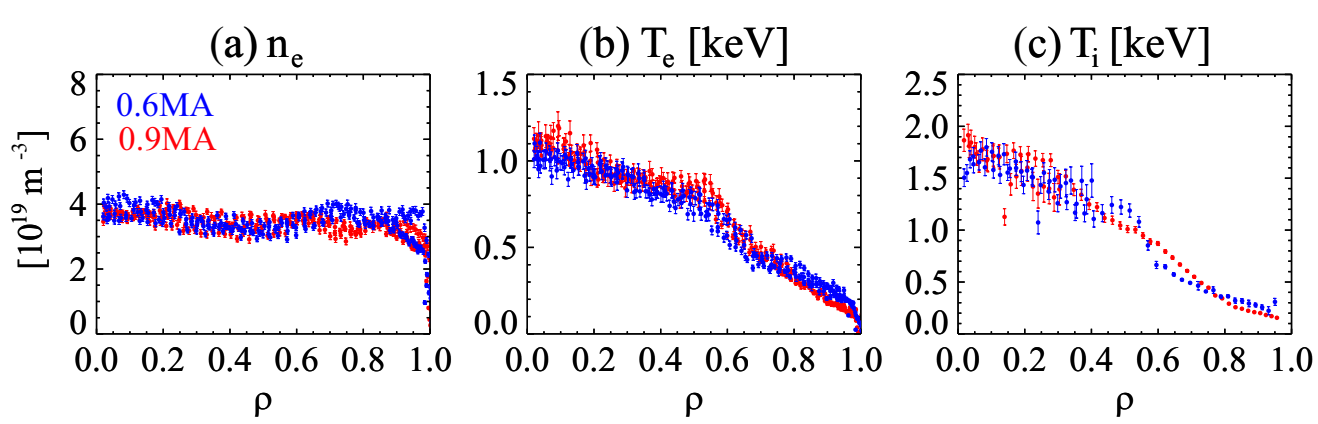

(d) $\mathrm{q}, \mathrm{q}_{\text {eng }}$

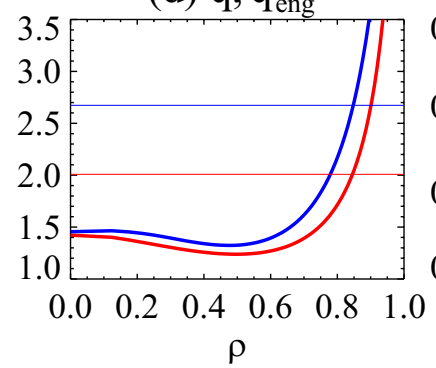

(e) $\mathrm{q}_{\mathrm{t}}, \mathrm{q}_{\mathrm{i}}, \mathrm{q}_{\mathrm{nc}}\left[\mathrm{MW} / \mathrm{m}^{2}\right]$

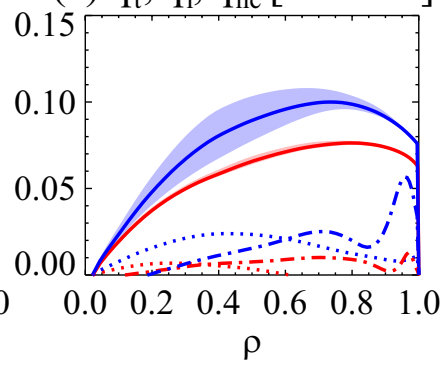

(f) $\mathrm{q}_{\mathrm{t}, 0.9 \mathrm{MA}} / \mathrm{q}_{\mathrm{t}, 0.6 \mathrm{MA}}$

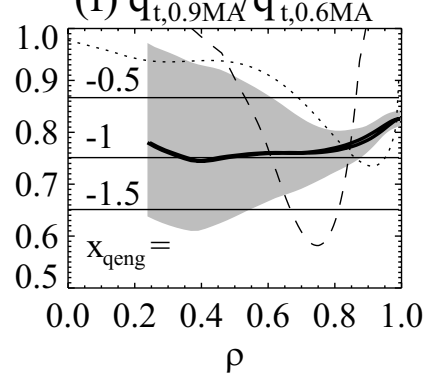

Figure 6. Profiles in the $q$ scan. Blue symbols: \#24206, $I_{\mathrm{p}}=0.62 \mathrm{MA}, P_{\mathrm{INJ}}=3.49 \mathrm{MW}$. Red symbols \#24207, $I_{\mathrm{p}}=0.91 \mathrm{MA}$, $P_{\mathrm{INJ}}=1.78 \mathrm{MW}$. Other notations as in figure 2. (d) Solid lines are the measured safety factor profiles, dotted lines are the values of global engineering safety factor $q_{\text {eng. }}$. $(f)$ Thick solid line: the ratio of total heat fluxes $q_{\mathrm{t}, 0.9 \mathrm{MA}} / q_{\mathrm{t}, 0.6 \mathrm{MA}}$, the shaded area is the uncertainty in $q_{\mathrm{t}, 0.9 \mathrm{MA}} / q_{\mathrm{t}, 0.6 \mathrm{MA}}$, solid horizontal lines indicate the values of exponent $x_{q}$ for given ratios of total heat flux; dashed line: ratio of electron heat diffusivities $\chi_{\mathrm{e}, 0.9 \mathrm{MA}} / \chi_{\mathrm{e}, 0.6 \mathrm{MA}}$, dotted line: the ratio of local safety factors $q_{0.9 \mathrm{MA}} / q_{0.6 \mathrm{MA}}$.

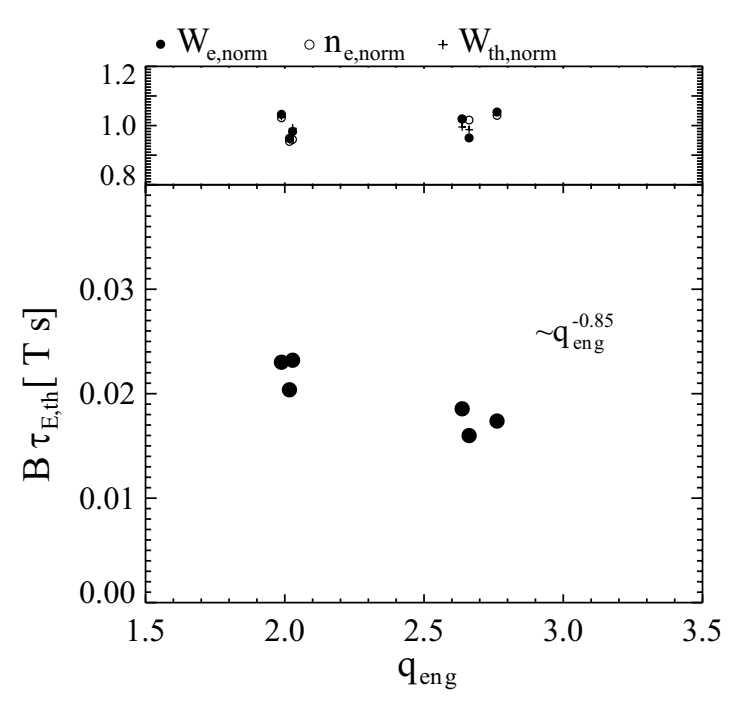

Figure 7. Main panel: safety factor scan of thermal energy confinement time. Top panel: variations of the electron energy content $W_{\mathrm{e}}$, line-averaged density $\bar{n}_{\mathrm{e}}$ and thermal energy $W_{\mathrm{th}}$, all normalized to average values along the scan.

The robustness of the two-point scan has been checked on a dataset of six observations, each representing one discharge. The top panel in figure 7 shows that the line-averaged density, electron and thermal energy contents are constant along the safety factor scan so that dimensionless parameters $\rho_{*}, \beta$ and $v_{*}$ are also constant. The main panel in figure 7 shows the values of thermal energy confinement time calculated by TRANSP. Log-linear regression on the dataset gives the scaling of $B \tau_{E \text {,th }} \propto q_{\mathrm{eng}}^{-0.85}$ with standard error in the exponent of $\delta x_{q}=0.2$. This value is consistent with the two-point scan described above.

\subsection{Effect of fast ion losses}

As mentioned above, anomalous fast ion losses in TRANSP are needed to match the measured neutron rate. For a high $q_{\text {eng }}$ plasma, which has high heating power, anomalous fast ion losses with diffusivity of $D_{\text {fast }}=(0.5-1) \mathrm{m}^{2} \mathrm{~s}^{-1}$ are needed to match the measured neutron rate. For a low $q_{\text {eng }}$ plasma, which also has a low heating power, fast ion losses with $D_{\text {fast }}=$ $(0-0.5) \mathrm{m}^{2} \mathrm{~s}^{-1}$ are sufficient to match the measured neutrons. This asymmetry means that inclusion of fast ion losses makes the safety factor scaling weaker. The scale of this effect has been evaluated by rerunning the two-point scan in figure 6 without anomalous fast ion losses for both plasmas. The ratio of power loss was found to be $P_{\mathrm{th}, 0.6 \mathrm{MA}} / P_{\mathrm{th}, 0.9 \mathrm{MA}}=1.45$ and the safety factor exponent $x_{q}=-1.32$. This value is about 2 standard errors larger than with fast ion losses included (figure 7).

The values of fast ion diffusivities $D_{\text {fast }}$ in the $q$ scan are lower than found in the collisionality scan. As mentioned above the physics of anomalous fast ion losses is outside the scope of this paper and will be investigated using newly installed diagnostics. Here we just note that in the $q$ scan the heating powers and electron temperatures are generally lower than in the $v_{*}$ scan. Both of these factors imply a smaller population of fast ions and consequently, a lower level of fast particle driven MHD activity which might be one of the factors responsible for anomalous fast ion diffusion, as discussed in section 3.3.

\section{Discussion}

The dimensionless scaling of energy confinement with safety factor and collisionality in MAST H-mode plasmas described 
above can be summarized as

$$
B \tau_{E, \text { th }} \propto \nu_{*}^{-0.82 \pm 0.1} q_{\mathrm{eng}}^{-0.85 \pm 0.2} .
$$

The dependence on collisionality is stronger than in the IPB98y 2 scaling, where $B \tau_{E \text {,th }} \propto v_{*}^{-0.01}$ [3]. The $v_{*}$ scaling (2) is similar to that reported from the NSTX [5]. It is also in line with dedicated scans performed on conventional tokamaks as summarized in review papers [20,21], where

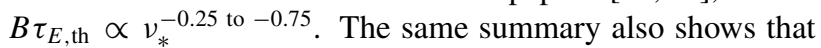
the $v_{*}$ exponents are correlated with collisionality itself in such a way that $v_{*}$ dependence is weaker at lower collisionality and vice versa (e.g. in low $\nu_{*}$ JET plasmas $B \tau_{E \text {,th }} \propto v_{*}^{-0.25}$ and in high $\nu_{*}$ CMOD plasmas $\left.B \tau_{E \text {,th }} \propto \nu_{*}^{-0.75}\right)$. It is reasonable to assume that a similar trend will exist for STs and therefore for extrapolation towards low $v_{*}$ one has to expect a weaker collisionality dependence than in scaling (2).

The safety factor dependence in (2) is different from that in the IPB98y 2 scaling, where $B \tau_{E \text {,th }} \propto q_{\text {eng }}^{-3.0}$ [3]. Our scaling is closer to the dedicated scans on DIII-D, where $B \tau_{E \text {,th }} \propto q_{\mathrm{eng}}^{-1.4}$ [22]. Note that our scan is similar to the scan with constant $q(0)$ rather than constant magnetic shear.

Dimensionless scaling (2) is consistent with a strong dependence of $\tau_{E \text {,th }}$ on toroidal magnetic field as reported in [4]. Transformation from dimensionless scaling (1) to engineering parameters $\tau_{E \text {,th }} \propto I_{\mathrm{p}}^{\alpha_{I}} B^{\alpha_{I}}$ shows that the sum of $I_{\mathrm{p}}$ and $B$ exponents is controlled mainly by $\nu_{*}$ scaling: the stronger the $v_{*}$ dependence, the larger the $\operatorname{sum} \alpha_{B}+\alpha_{I}=\left(-x_{\rho}-1-2 x_{\beta}\right) /\left(1-0.5 x_{\rho}-x_{\beta}+2 x_{v}\right)$. The partition between current and toroidal field exponents is mainly controlled by $q_{\text {eng }}$ scaling: the weaker the $q_{\text {eng }}$ scaling, the stronger the $B$ dependence, $\alpha_{B} /\left(\alpha_{B}+\alpha_{I}\right)=$ $1+x_{q} /\left(-x_{\rho}-1-2 x_{\beta}\right)$. If $\rho_{*}$ and $\beta$ dependences are assumed in a plausible range, $B \tau_{E \text {,th }} \propto \rho_{*}^{-3}$ to $-2.5 \beta^{-0.5 \text { to } 0}$ [20], the scaling (2) translated into toroidal field dependence gives $\tau_{E \text {,th }} \propto B^{1.1}$ to 1.6 . This is in good agreement with our toroidal field scan in [4].

Although the scalings with all dimensionless parameters are not yet measured in STs, some conclusions can be drawn already from formula (2) as described below.

\subsection{Prediction to ST-CTF}

The parameters of the ST-CTF device listed in section 2 have been determined using the IPB98y 2 confinement scaling in which $\tau_{E \text {,th }}^{\text {IPB } 982} B \propto q_{\text {eng }}^{-3.0} \nu_{* q}^{-0.01} \kappa^{3.3}$ [3] (note the correction for $\kappa$ exponent in [23]). This is in contrast to the MAST data and therefore it is of interest to analyse the difference in prediction if scaling (2) is used instead. Consider first the gap between MAST low collisionality reference shot \#22664 from table 1 and the nominal ST-CTF operation point. The ratio of dimensionless energy confinement time between these two plasmas is

$$
\left(\tau_{E, \mathrm{th}} B\right)_{\mathrm{CTF}} /\left(\tau_{E, \mathrm{th}} B\right)_{22664}=11 .
$$

According to IPB98y 2 scaling one half of this extrapolation comes from three variables: $q_{\mathrm{eng}}, \nu_{* q}$ and $\kappa$ :

$$
\left(q_{\text {eng }}^{-3.0} v_{* q}^{-0.01} \kappa^{3.3}\right)_{\mathrm{CTF}} /\left(q_{\text {eng }}^{-3.0} v_{* q}^{-0.01} \kappa^{3.3}\right)_{22664}=5.9 .
$$

Let us consider scaling (2) with no elongation dependence: $\tau_{E, \text { th }} B \propto q_{\text {eng }}^{-0.85} v_{*}^{x_{v}} \kappa^{0}$. It can be shown that this scaling provides the same confinement multiplier as in equation (3) if the collisionality dependence is $x_{v}=-0.38$. This shows that weaker $q_{\text {eng }}$ scaling in (2) can be compensated by stronger $v_{*}$ dependence.

IPB98 $y 2$ scaling with its strong $q_{\text {eng }}$ dependence favours high plasma current operation. Because in the IPB98 2 scaling the explicit contribution from elongation is small, $\tau_{E, \text { th }}^{\text {IPB }}{ }^{2} B \propto$ $q_{\text {eng }}^{-3.0} \kappa^{3.3} \propto\left(I_{\mathrm{p}} / B\right)^{3.0} \kappa^{0.3}$, a large part of the confinement multiplier (3) is due to the strong dependence on $I_{\mathrm{p}} / B$. Indeed for the ST-CTF, the value $I_{\mathrm{p}} / B$ has been set 1.5 larger than in the MAST reference \#22664 shot. Attempts to run plasmas with such high $I_{\mathrm{p}} / B$ were accompanied with control difficulties so far, mainly due to low ELM frequency. Scaling (2), however, opens the possibility to reduce the current in the ST-CTF while keeping the confinement multiplier unchanged. The allowable reduction in plasma current depends on the collisionality scaling. For instance, if $\tau_{E, \text { th }} B \propto q_{\text {eng }}^{-0.85} \nu_{*}^{-0.47} \kappa^{0}$ the plasma current can be reduced by a factor of 1.5 so that $I_{\mathrm{p}} / B$ is the same as in the MAST reference shot. Reduction in plasma current will also reduce the required neutral beam power, mainly set by current drive, and thus the whole operation point for ST-CTF will need reassessment.

\subsection{Upgrade of MAST}

A planned upgrade of MAST includes an increase in the magnetic field $B$ by a factor of 1.5 and heating power $P_{\mathrm{L}, \mathrm{th}}$ by a factor of 2 from the values of reference shot \#22664. This could allow the reduction of the collisionality $v_{*} \propto B^{-4}$ by a factor of 5 . Projecting from the reference shot such a plasma should have central electron temperature $T_{\mathrm{e}, 0}=$ $T_{\mathrm{e}, 0}(\# 22664) \times 1.5^{2}=3 \mathrm{keV}$. Whether such temperatures can be achieved with the available power will depend on the collisionality scaling. The required power depends on collisionality as $P_{\mathrm{L}, \mathrm{th}} \propto \nu_{*}^{-x_{v}-3 / 4}$ so that the factor of 2 power increase restricts the scaling exponent as $(1 / 5)^{-x_{v}-3 / 4}<2$. This condition is satisfied if the collisionality scaling will be stronger than $x_{v}<-0.3$.

\section{Conclusions}

Dimensionless confinement scalings with collisionality and safety factor measured in MAST H-mode show favourable trends for extrapolation towards future spherical tokamaks. Relatively weak safety factor scaling places more emphasis on the increase in the toroidal magnetic field rather than plasma current. Strong collisionality scaling is responsible for a strong dependence of confinement time on toroidal magnetic field. This link, however, also means that such a magnetic field scaling is expected only in the region of strong $v_{*}$ dependence. Therefore, future experiments in MAST should search for a sign for weakening of the collisionality dependence as plasmas are extended to lower and lower values of $v_{*}$. In addition, further validation of fast ion energy transport is important for reducing scaling uncertainties. Finally, dependences on the remaining dimensionless parameters in equation (1) should be established to improve the physics basis for devices such as the ST-CTF. 


\section{Acknowledgments}

This work was funded by the RCUK Energy Programme under grant EP/I501045 and the European Communities under the contract of Association between EURATOM and CCFE. The views and opinions expressed herein do not necessarily reflect those of the European Commission. The authors would like to thank Drs B. Lloyd and A.W. Morris for valuable comments. Anonymous referees are gratefully acknowledged for their suggestions.

\section{(C) Euratom 2011.}

\section{References}

[1] Voss G.M. et al 2008 Fusion Eng. Des. 831648

[2] European Commission. 'R\&D Needs and Required Facilities for the Development of Fusion as an Energy Source' October 2008, http://ec.europa.eu/research/ energy/pdf/978-92-79-10057-4_en.pdf

[3] ITER Physics Basis Expert Groups on Confinement and Transport and Confinement Modelling and Database, ITER Physics Basis Editors 1999 Nucl. Fusion 392175
[4] Valovič M. et al 2009 Nucl. Fusion 49075016

[5] Kaye S. et al 2006 Nucl. Fusion 46848

[6] Kaye S. et al 2007 Nucl. Fusion 47499

[7] Valovič M. et al 2005 Nucl. Fusion 45942

[8] Kaye S. et al 2006 Plasma Phys. Control. Fusion 48 A 429

[9] Petty C.C. and Luce T.C. 1999 Phys. Plasmas 6909

[10] Candy J. and Waltz R.E. 2003 J. Comput. Phys. 186545

[11] Kotschenreuther M. et al 1995 Comput. Phys. Commun. 88128

[12] Applegate D.J. et al 2007 Plasma Phys. Control. Fusion 491113

[13] Wong K.L. et al 2007 Phys. Rev. Lett. 99135003

[14] Guttenfelder W. et al 2011 Phys. Rev. Lett. 106155004

[15] Redd A.J. et al 1999 Phys. Plasmas 61162

[16] Roach C.M. et al 2009 Plasma Phys. Control. Fusion 51124020

[17] Guttenfelder W. and Candy J. 2011 Phys. Plasmas 18022506

[18] Smith D.R. et al 2009 Phys. Rev. Lett 102225005

[19] Gorelenkov N.N. et al 2010 Nucl. Fusion 50084012

[20] Luce T.C., Petty C.C. and Cordey J.G. 2008 Plasma Phys. Control. Fusion $\mathbf{5 0} 043001$

[21] Petty C.C. 2008 Phys. Plasmas 15080501

[22] Petty C.C. et al 1998 Phys. Plasmas 51695

[23] McDonald D.C. et al 2007 Nucl. Fusion 47147 